\title{
WETTING ON ROUGH SURFACES AND CONTACT ANGLE HYSTERESIS: NUMERICAL EXPERIMENTS BASED ON A PHASE FIELD MODEL
}

\author{
Alessandro Turco ${ }^{1}$, François Alouges $^{2}$ And Antonio DeSimone ${ }^{1}$
}

\begin{abstract}
We present a phase field approach to wetting problems, related to the minimization of capillary energy. We discuss in detail both the $\Gamma$-convergence results on which our numerical algorithm are based, and numerical implementation. Two possible choices of boundary conditions, needed to recover Young's law for the contact angle, are presented. We also consider an extension of the classical theory of capillarity, in which the introduction of a dissipation mechanism can explain and predict the hysteresis of the contact angle. We illustrate the performance of the model by reproducing numerically a broad spectrum of experimental results: advancing and receding drops, drops on inclined planes and superhydrophobic surfaces.
\end{abstract}

Mathematics Subject Classification. 76D45, 74N30, 49S05.

Received February 1st, 2008. Revised November 24, 2008.

Published online June 12, 2009.

\section{INTRODUCTION}

We consider the problem of finding the equilibrium shape of a liquid drop surrounded by a fluid (either vapor or another liquid immiscible with that of the drop) and in contact with a solid surface when the solid support has a nontrivial shape. The macroscopic physics governing this problem is quite simple: the shape is the one that minimizes the total interfacial energy at fixed drop volume. If the solid surface is flat, the solution is well known to be a spherical cap, with contact between liquid and solid in the shape of a circular disk, and a contact angle given by Young's law $[9,11]$.

If the solid surface is patterned at a microscopic scale, either chemically or because of asperities, then the geometric characterization of the solid-liquid contact is not trivial. Different models have been proposed. Some are phenomenological, like the Cassie-Baxter and the Wenzel ones [9], which are based on different hypotheses on the microscopic geometry of the solid-liquid interface (uniform and complete contact between solid and liquid in the Wenzel case, composite contact allowing for pockets of vapour trapped between solid and liquid in the Cassie-Baxter scenario). Other proposals are more mathematically based [1,15]. In fact, it has been shown in [1] using the techniques of homogenization theory, that both the Wenzel and the Cassie-Baxter structures may emerge as the one minimizing the interfacial energy, depending on the chemical and topographical details of the solid substrate.

\footnotetext{
Keywords and phrases. Wetting, contact angle hysteresis, super-hydrophobic surfaces.

1 Scuola Internazionale Superiore di Studi Avanzati, Via Beirut 2, 34014 Trieste, Italy. desimone@sissa.it

2 Université Paris XI, 91405 Orsay Cedex, France.
} 


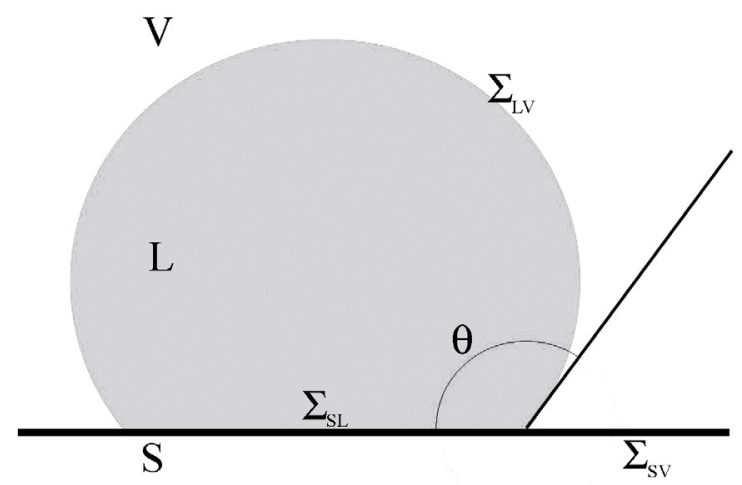

FIGURE 1. The equilibrium shape of a liquid drop $(L)$ surrounded by vapor $(V)$ and placed over a flat solid surface $(S)$ is a spherical cap. The contact angle $\theta$ is determined by the physical parameters of the problem (surface tensions).

Furthermore if we look at the evolution of drops driven by changing their volume or by tilting the solid support quasi-statically, other features emerge: for example the contact angle is different when the front advances or when it recedes [9]. A first attempt to derive macroscopic models for contact angle hysteresis by applying homogenization techniques to the microscopic laws of capillarity is in [10], but much remains to be done. In this paper, we propose a phase field model to reproduce numerically the available experimental evidence.

Within a phase field approach, the geometry of the drop is described by using a phase function $\phi$ that takes the value 1 in the liquid phase, the value 0 in the environmental fluid, and spans the whole $[0,1]$ interval in a liquid-vapor transition region. Thus the sharp interfaces of the (geometric) capillary model are replaced by narrow transition layers of width of order $\epsilon>0$, a small parameter. The equilibrium shape of the drop is obtained by setting up a steepest descent dynamics for $\phi$, which then tends to a state minimizing an $\epsilon$-regularized version of the capillary energy. The parabolic PDE we obtain resembles the classical Allen-Cahn equation and its general form reads:

$$
-\epsilon \triangle \phi+\frac{1}{\epsilon} \phi(1-\phi)(1-2 \phi)+\lambda=0
$$

In the limit as $\epsilon$ tends to 0 , one recovers the solution of the capillary problem, with sharp interfaces between the phases. The presence of the solid is modeled by imposing suitable boundary condition to the phase field function (both Neumann and Dirichlet conditions have been implemented). The model can be easily adapted to reproduce contact angle hysteresis, by changing the boundary conditions in order to account for the pinning effects on the contact line due to dissipation.

The rest of the paper is organized as follows. In Sections 2 and 3 we discuss the models in detail. In particular, we discuss the relationship between the geometric and phase field formulation for the problem of finding global minimizers for the capillarity energy. Section 4 is devoted to the modeling of contact angle hysteresis in the quasi-static evolution of capillary drops. In Section 5 we give the details of our proposed numerical method. Section 6 contains the description of some numerical simulations reproducing known (and striking!) laboratory experiments.

\section{Phase Field MOdel FOR CAPIllary DROPS}

The energy of a liquid drop $\omega$ in contact with a solid $S$ and surrounded by a fluid (usually air, so we will refer to it as vapor; but we can also consider the case of two immiscible fluids, like drops of oil in water) is [9,11]

$$
E(\omega, t)=\int_{\partial_{S} \omega} \sigma_{S L}(x) \mathrm{d} A_{x}+\sigma_{L V} \int_{\partial_{V} \omega} \mathrm{d} A_{x}+\int_{\partial S \backslash \partial_{S} \omega} \sigma_{S V}(x) \mathrm{d} A_{x}+\int_{\omega} \rho_{L}(x) G(x, t) \mathrm{d} \mathcal{V}_{x}
$$


where $\partial_{S} \omega=\partial \omega \cap \partial S$ is the interface between liquid and solid, often called $\Sigma_{S L} ; \partial_{V} \omega=\partial \omega \backslash \partial_{S} \omega$ is the liquid-vapor interface $\Sigma_{L V} ; \partial S \backslash \partial \omega$ is the solid-vapor one, $\Sigma_{S V} ; \rho_{L}$ represents the density of the fluid (we will always consider a homogeneous fluid and we will set $\rho_{L}=1$ ) and $G(x, t)$ is a generic potential related to an external force field (gravity, for example). The terms $\sigma_{A B}(x)$ are the surface energies (or surface tensions) at a point $x$ on the $A B$ interface. In the case where $S$ is a homogeneous solid, these are constant and the energy becomes

$$
\begin{aligned}
E(\omega, t) & =\sigma_{S L}\left|\Sigma_{S L}\right|+\sigma_{L V}\left|\Sigma_{L V}\right|+\sigma_{S V}\left|\Sigma_{S V}\right|+\int_{\omega} G(x, t) \mathrm{d} \mathcal{V}_{x} \\
& =\left(\sigma_{S L}-\sigma_{S V}\right)\left|\Sigma_{S} L\right|+\sigma_{L V}\left|\Sigma_{L V}\right|+\int_{\omega} G(x, t) \mathrm{d} \mathcal{V}_{x}+k
\end{aligned}
$$

where $|A|$ denotes the measure of the set $A$ and $k$ is a constant that does not enter in the search for the minima of the functional, and so it will be omitted.

The problem of capillarity is:

$$
\text { Given a volume } \mathcal{V}>0 \text {, find } \omega^{*}=\underset{|\omega|=\mathcal{V}}{\operatorname{argmin}}\{E(\omega, t)\} .
$$

In some cases, it is of interest to consider an evolutionary problem driven for example by the time evolution of the volume constraint, according to some law of the type $t \mapsto \mathcal{V}(t)$ so that $\omega^{*}$ will be a function of the parameter $t$. The Euler-Lagrange equations for this problem are classical [11] and give the Laplace law

$$
2 H_{\Sigma_{L V}}(x)=\frac{p}{\sigma_{L V}}+\frac{1}{\sigma_{L V}} G(x, t)
$$

where $H_{\Sigma_{L V}}(x)$ is the mean curvature of $\Sigma_{L V}$ at $x$ and $p$ is the Lagrange multiplier associated with the volume constraint (its physical meaning is the jump of pressure across $\Sigma_{L V}$ ). The Young condition on the contact angle $\theta$ of the solid-liquid interface, see Figure 1, is also recovered as a necessary condition for (2.1) to be stationary

$$
\cos \theta=-\frac{\sigma_{S L}-\sigma_{S V}}{\sigma_{L V}}=: \cos \theta^{Y} .
$$

A phase field formulation for the capillarity problem is obtained by considering an energy of the type

$$
E_{\epsilon}(\phi, t)=\int_{\Omega} \epsilon|\nabla \phi|^{2}+\frac{1}{\epsilon} W(\phi)+\phi G(x, t) \mathrm{d} \mathcal{V}_{x}
$$

where $\phi$ is the phase function. The non-negative potential $W(\phi)$ vanishes only for the values of $\phi$ representing the vapor and the liquid phases. It is tuned in order to produce the correct interfacial surface tension values from the corresponding interphase transition layers in the limit as $\epsilon \rightarrow 0$. Our analysis rests on a slight modification of results by Baldo and Bellettini [4] and Modica [13], but there is a large mathematical literature on this topic starting from the ideas of Modica and Mortola [14]. We consider two alternative formulations, one based on Dirichlet boundary conditions, the other one based on Neumann boundary conditions. For the former case we collect all the results we need in the following theorem. We will deal with Neumann conditions in Section 3.

For any fixed $t$ (from here onwards, we will simplify our notation by leaving the dependence on $t$ in the functionals (2.1) and (2.6) as tacitly understood), we define

$$
\begin{aligned}
& \bar{E}_{\epsilon}(\phi)= \begin{cases}E_{\epsilon}(\phi) & \text { if } \phi \in H^{1}\left(\Omega, \mathbb{R}^{2}\right) \text { and }\left.\phi\right|_{\partial \Omega}=g, \\
+\infty & \text { otherwise in } L^{1} ;\end{cases} \\
& \bar{E}_{0}(\phi)= \begin{cases}E(\{\phi \equiv L\}) & \text { if } \phi \in B V(\Omega,\{V, L\}), \\
+\infty & \text { otherwise in } L^{1}\end{cases}
\end{aligned}
$$


where $g$ is a suitable function to be specified later (see (2.12)), while $V, L \in \mathbb{R}^{2}$ are two pairs of values for the phase function defining the vapor and the liquid phases. We will prove that if $\phi_{\epsilon}^{*}$ is a family of minimizers of $\bar{E}_{\epsilon}$ and if $\phi^{*}$ is its limit in $L^{1}$ (the existence of the limit is part of the proof), then the first component of $\phi^{*}$ is the characteristic function of a solution for the capillary problem (2.3), while the second one is 0 everywhere.

The external force term does not depend explicitly on $\epsilon$ (see Eq. (2.6)) and we can treat it separately. Indeed, by standard properties of $\Gamma$-convergence [8], we have only to check its continuity in the desired topology. In our case $\phi \mapsto \int \phi G \mathrm{~d} \mathcal{V}_{x}$ is continuous for the $L^{1}$ topology if $G$ is regular enough. The gravitational potential, for example, is admissible. Thus, in the theorem, we can assume $G \equiv 0$ for simplicity of exposition.

We set the problem in $\Omega$, a bounded subset of $\mathbb{R}^{n}$ (for us $n=2,3$ ) with piecewise $\mathcal{C}^{2}$ and Lipschitz boundary. We decompose it into two parts: $\partial \Omega=\partial_{S} \Omega \cup \partial_{V} \Omega$ (the set $\partial_{S} \Omega$ coincides exactly with what we called $\partial S$ in $(2.1))$. For $\psi=\left(\psi_{1}, \psi_{2}\right) \in \mathbb{R}^{2}$, the potential is

$$
W(\psi)=a^{2} \psi_{1}^{2}\left(1-\psi_{1}\right)^{2}+b^{2} \psi_{2}^{2},
$$

where

$$
\begin{aligned}
& a=3 \sigma_{L V}>0, \\
& b=\frac{1}{2}\left(\sigma_{S V}+\sigma_{S L}-\sigma_{L V}\right)>0 .
\end{aligned}
$$

We denote by $L=(1,0)$ and $V=(0,0)$ the only two zeros of $W$, as announced. Finally, let $g: \partial \Omega \rightarrow \mathbb{R}^{2}$ be such that $g \equiv V$ on $\partial_{V} \Omega$ and $g \equiv\left(\phi_{S}, 1\right):=S$ on $\partial_{S} \Omega$, where $\phi_{S}$ is the unique solution of

$$
\cos \theta^{Y}=-4 \phi_{S}^{3}+6 \phi_{S}^{2}-1 \quad 0 \leq \phi_{S} \leq 1 .
$$

We are now in position to state the following theorem.

Theorem 2.1. Let $\bar{E}_{0}$ and $\bar{E}_{\epsilon}$ be given by (2.8) and (2.7). Then $\bar{E}_{0}$ is the $\Gamma$-limit of $\bar{E}_{\epsilon}$ as $\epsilon$ tends to zero in the topology of $L^{1}$. Moreover for every $\epsilon>0$ let $\phi_{\epsilon}^{*}=\operatorname{argmin}\left\{\bar{E}_{\epsilon}(\phi): \int_{\Omega} \phi_{1}=\mathcal{V}\right\}$; then the sequence $\left(\phi_{\epsilon}^{*}\right)$ is pre-compact in $L^{1}$ and every cluster point, say $\phi^{*}$, belongs to $B V(\Omega,\{V, L\})$ and we have $\phi^{*}=\operatorname{argmin}\left\{\bar{E}_{0}(\phi): \int_{\Omega} \phi_{1}=\mathcal{V}\right\}$.

Proof. The proof uses classical arguments, but we give here some details because they are essential in the construction of the numerical scheme presented in Section 5. Our discussion entails two steps: in the first one we establish the link between our problem and that considered by Baldo and Bellettini [4]. After that we show how to handle the volume constraint.

Let us define a metric on $\mathbb{R}^{2}$ (other possible and equivalent definitions of this metric are presented in [4], this formulation is the computationally most convenient one):

$$
d\left(v_{1}, v_{2}\right)=\min \left\{\int_{-\infty}^{+\infty}\left\{\dot{\rho}^{2}+W(\rho)\right\} \mathrm{d} t \quad \rho(-\infty)=v_{1}, \rho(+\infty)=v_{2}\right\}
$$

and a functional on $B V(\Omega,\{V, L\})$

$$
\tilde{E}_{0}(\phi)=2 d(V, L) \mathcal{H}_{n-1}\left(\partial^{*}\{\phi \equiv L\} \cap \partial^{*}\{\phi \equiv V\}\right)+2 \int_{\partial \Omega} \mathrm{d}\left(\left.\phi\right|_{\partial \Omega}(x), g(x)\right) \mathrm{d} \mathcal{H}_{n-1}(x)
$$

where $\partial^{*} A$ denotes the reduced boundary of the set $A$ and $\mathcal{H}_{n-1}$ denotes the Hausdorff measure of dimension $n-1$. We now want to show that in our situation $2 \bar{E}_{0}=\tilde{E}_{0}$. In the wetting setting, we label as "liquid" the set $\{\phi \equiv L\}$ and the set $\{\phi \equiv V\}$ as "vapor". Moreover, considering our choice of $g$, the functional (2.14) becomes

$$
\tilde{E}_{0}(\phi)=2 d(V, L)\left|\Sigma_{L V}(\phi)\right|+2 d(V, S)\left|\Sigma_{S V}(\phi)\right|+2 d(S, L)\left|\Sigma_{S L}(\phi)\right| .
$$


Indeed, the integral in (2.14) decomposes into the sum of two terms because $g$ is constant and $\phi$ can assume only the values $V$ and $L$. Then we have only to show that equation (2.12) and the metric (2.13) give us the correct value for the surface tensions if we choose appropriately the values of the parameters $a$ and $b$ in the potential. Substituting the appropriate values, we obtain

$$
\begin{aligned}
\sigma_{L V}=d(L, V) & =\min \int_{-\infty}^{+\infty}\left\{\dot{\rho}_{1}^{2}+W\left(\left(\rho_{1}, 0\right)\right)\right\} \mathrm{d} t \\
& =2 \int_{0}^{1} \sqrt{W((\tau, 0))} \mathrm{d} \tau=\frac{a}{3} \\
\sigma_{S V}=d(S, V) & =2 \int_{0}^{\phi_{S}} \sqrt{W((\tau, 0))} \mathrm{d} \tau+2 \int_{0}^{1} \sqrt{W((0, \tau))} \mathrm{d} \tau \\
& =-2 a\left(-\frac{\phi_{S}^{2}}{2}+\frac{\phi_{S}^{3}}{3}\right)+b \\
\sigma_{S L}=d(S, L) & =2 \int_{\phi_{S}}^{1} \sqrt{W((\tau, 0))} \mathrm{d} \tau+2 \int_{0}^{1} \sqrt{W((0, \tau))} \mathrm{d} \tau \\
& =2 a\left(\frac{1}{6}-\frac{\phi_{S}^{2}}{2}+\frac{\phi_{S}^{3}}{3}\right)+b .
\end{aligned}
$$

Hence $b=\frac{1}{2}\left(\sigma_{S L}+\sigma_{S V}-\sigma_{L V}\right)$ and equation (2.12) holds. Notice that the condition $b>0$ can always be reached because we can add the same quantity to $\sigma_{S V}$ and $\sigma_{S L}$ without changing the problem: the system (2.16)-(2.18) sees only the difference $\sigma_{S V}-\sigma_{S L}$. Notice also that a key feature of our approach is to choose a value for the boundary condition that is not a zero of the potential: the metric $d$ measures the distance between $\phi$ and the value at the boundary and it reproduces the correct interfacial energy. Finally, observe that the form of $W$, see (2.9), implies that the minimal $\phi_{2}$ is always the constant 0 , also if this value does not match the boundary condition. Thus, from now on, we will write simply $\phi$ instead of $\phi_{1}$ to denote the only nontrivial component of the phase function.

Having established the link with the setting of [4], the proof of the $\Gamma$-convergence result can be easily adapted to our case. Handling the volume constraint is not a difficult task $[3,6,8]$. The subspace $\left\{\phi \in L^{1}\right.$, $\left.\int \phi_{1}=\mathcal{V}\right\}$ is closed in the $L^{1}$ topology and, in the recovering sequence of the $\Gamma$-lim sup, we can always assume $\int \phi_{\epsilon}=\int \phi$.

The external force field can be easily added at this point. From the theorem we know that when $\epsilon$ goes to zero the minimizers of $\bar{E}_{0}$ are functions in $B V(\Omega,\{V, L\})$. More precisely the first component will take the value 1 in the region occupied by the liquid and the value 0 in the vapor region. Hence, looking at (2.2) and (2.6),

$$
\int_{\Omega} \phi_{1} G(x, t) \mathrm{d} \mathcal{V}_{x}=\int_{\omega} G(x, t) \mathrm{d} \mathcal{V}_{x}
$$

as desired.

\section{NEUMANN BOUNDARY CONDITIONS}

We now describe an alternative approach to the phase field formulation, based on Neumann-type boundary conditions. For this purpose, we use a result of Modica [13]. We consider, for simplicity, the situation of a region $\Omega \subseteq \mathbb{R}^{3}$ whose boundary is the solid surface $S$. Inside this set we want to solve the capillary problem for a prescribed volume of liquid $L$. In the sequel we will not discuss explicitly the volume constraint, which can be added later exactly as before. Our formulation is based on a potential of the type $W(x)=a^{2} x^{2}(1-x)^{2}$ (with $a>0$ to be specified later), while in [13] the minima of the potential should be strictly positive: this limitation can be removed by choosing appropriately the boundary term (see the functional (3.3) below). 
Let $\sigma:[0 ;+\infty) \rightarrow \mathbb{R}^{+}$be any continuous function and define

$$
\begin{gathered}
\hat{\sigma}(x)=\inf _{s \geq 0}\left\{\sigma(s)+2\left|\int_{x}^{s} \sqrt{W(y)} \mathrm{d} y\right|\right\}, \\
c_{0}=\int_{0}^{1} \sqrt{W(y)} \mathrm{d} y .
\end{gathered}
$$

Consider the functional

$$
E_{\epsilon}^{N}(\phi)= \begin{cases}\int_{\Omega} \epsilon\left|D \phi_{\epsilon}\right|^{2}+\frac{1}{\epsilon} W\left(\phi_{\epsilon}\right) \mathrm{d} x+\int_{\partial \Omega} \sigma\left(\tilde{\phi}_{\epsilon}\right) \mathrm{d} \mathcal{H}_{n-1}(x) & \text { if } \phi \in H^{1}(\Omega, \mathbb{R}), \\ +\infty & \text { otherwise in } L^{1},\end{cases}
$$

where $\tilde{\phi}_{\epsilon}$ denotes the trace of $\phi_{\epsilon}$ on the boundary. Then we have that $E_{\epsilon}^{N} \Gamma$-converges to [13]

$$
E_{0}^{N}(\phi)=2 c_{0}\left|\Sigma_{L V}\right|+\hat{\sigma}(1)\left|\Sigma_{S L}\right|+\hat{\sigma}(0)\left|\Sigma_{S V}\right| .
$$

The physical interpretation of the convergence result is the following. Choose, as in [17], $\sigma(x):=N x$, where $N$ is a constant. The Euler-Lagrange equations for (3.3) yields the boundary condition

$$
-2 \epsilon \frac{\partial \phi}{\partial n}=N
$$

Here $n$ is the outward unit normal vector to $\partial \Omega$. By an appropriate choice of $a$ and $N$ we can recover the correct surface tensions. This is an easy calculation:

$$
\begin{gathered}
2 c_{0}=\frac{a}{3}=\sigma_{L V}, \\
\hat{\sigma}(0)=0, \\
\hat{\sigma}(1)=\inf _{s \geq 0}\left\{N s+2 a\left(\frac{s^{3}}{3}-\frac{s^{2}}{2}+\frac{1}{6}\right)\right\}=\sigma_{S L}-\sigma_{S V} .
\end{gathered}
$$

We remark that the hypothesis on the sign of the function $\sigma(\cdot)$, restricts our study to values $N \geq 0$ and thus to contact angles $\theta^{Y} \geq \frac{\pi}{2}$. The model for acute contact angles can be obtained by putting $\phi \equiv 0$ in the liquid phase and $\phi \equiv 1$ in the vapor. It is easy to see that in the Euler-Lagrange equation this exchange produces the same results than considering negative values of $N$ in the usual representation. In any case, the study of the minimum problem (3.8) gives us a non-linear equation that chooses the right value of $N$ for the angle we want to simulate.

\section{Contact Angle hysteresis: inCREMEntal Formulation FOR QUASISTATIC EVOLUTION}

Following $[2,10]$, we consider the following discrete incremental formulation for the problem of the quasistatic evolution of a drop. Given the configuration $\omega^{*}(t)$ of a drop at time $t$, the one at time $t+\delta t$ is given by:

$$
\omega^{*}(t+\delta t)=\underset{|\omega|=\mathcal{V}(t+\delta t)}{\operatorname{argmin}}\left\{E(\omega, t+\delta t)+D\left(\omega, \omega^{*}(t)\right)\right\}
$$

where the dissipation $D\left(\omega_{1}, \omega_{2}\right)$ is given by

$$
D\left(\omega_{1}, \omega_{2}\right)=\mu\left|\partial_{S} \omega_{1} \triangle \partial_{S} \omega_{2}\right| .
$$


Here $A \triangle B=(A \backslash B) \cup(B \backslash A)$ denotes the symmetric difference of the sets $A$ and $B$ and $\mu>0$ is a parameter giving the dissipated energy per unit variation of the wetted area.

To illustrate the meaning of this formulation, we consider the case of a drop on a horizontal plane, subject to no gravity. It can be shown that, in this case, $\omega^{*}(t)$ is always a spherical cap [11]. Thus energy and dissipation can be written as:

$$
\begin{gathered}
E=\left(\sigma_{S L}-\sigma_{S V}\right) \pi a^{2}+\sigma_{L V} A \\
D\left(\omega_{1}, \omega_{2}\right)=D\left(a_{1}, a_{2}\right)=\mu \pi\left|a_{1}^{2}-a_{2}^{2}\right|
\end{gathered}
$$

where $A=2 \pi R h$ is the area of the spherical cap of radius $R$ and height $h$, while $a$ is the radius of the wetted area, namely, the interface between the solid and the liquid. Since we have that

$$
\left.\delta A\right|_{|\omega|=\mathcal{V}(t+\delta t)}=2 \pi a \cos \theta \delta a,
$$

where the left hand side denotes variations of $A$ at fixed volume $|\omega|=\mathcal{V}(t+\delta t)$, the Euler-Lagrange equation for the incremental variational problem becomes

$$
-\left(\sigma_{S L}-\sigma_{S V}\right) 2 \pi a-\sigma_{L V} \cos \theta 2 \pi a \in \partial \mu \pi\left|a^{2}-a^{2}(t)\right|,
$$

where

$$
\partial \mu \pi\left|a^{2}-a^{2}(t)\right|= \begin{cases}\{2 \pi a \mu\} & \text { if } a>a(t) \\ \pi a[-\mu, \mu] & \text { if } a=a(t) \\ \{-2 \pi a \mu\} & \text { if } a<a(t)\end{cases}
$$

is the sub-differential of the convex function $a \mapsto \mu \pi\left|a^{2}-a^{2}(t)\right|$. Another way to see this is to consider left and right variation for the parameter $a$. In this case we do not have to face the singularity of the dissipation term (4.4) at $a_{1}=a_{2}$ and we will deduce a couple of inequalities. In any case, the result is the following

$$
\cos \theta \in \begin{cases}\left\{\cos \theta^{r}\right\} & \text { if } a<a(t) \\ {\left[\cos \theta^{r}, \cos \theta^{a}\right]} & \text { if } a=a(t) \\ \left\{\cos \theta^{a}\right\} & \text { if } a>a(t)\end{cases}
$$

where

defines the advancing contact angle and

$$
\cos \theta^{a}=\cos \theta^{Y}-\frac{\mu}{\sigma_{L V}}
$$

$$
\cos \theta^{r}=\cos \theta^{Y}+\frac{\mu}{\sigma L V}
$$

defines the receding contact angle.

Returning to the general case (4.1), we look for solutions $\omega=\omega(t+\delta t)$ of the incremental problem: minimize

$$
F(\omega, t+\delta t)=\left(\sigma_{S L}-\sigma_{S V}\right)\left|\partial_{S} \omega\right|+\sigma_{L V}\left|\partial_{V} \omega\right|+\mu\left|\partial_{S} \omega \Delta \partial_{S} \omega(t)\right|
$$

by the phase field method discussed in Section 2. We can also add the gravity term, if needed. Thus, in practice, we are led to solve

$$
\phi_{\epsilon}^{*}(t+\delta t)=\operatorname{argmin}\left\{E_{\epsilon}(\phi, t+\delta t), \text { subject to } \int_{\Omega} \phi=\mathcal{V}(t+\delta t)\right\}
$$


with

$$
\phi= \begin{cases}\phi_{S}^{a} & \text { on } \partial \Omega_{\epsilon}^{a} \\ \phi_{S}^{r} & \text { on } \partial \Omega_{\epsilon}^{r}\end{cases}
$$

where $\phi_{S}^{a}$ and $\phi_{S}^{r}$ are the Dirichlet boundary conditions associated with the advancing and the receding angle respectively, computed with an equation similar to that in (2.12), but with $\theta^{a}, \theta^{r}$ in place of $\theta^{Y}$. The regions $\partial \Omega_{\epsilon}^{r}$ and $\partial \Omega_{\epsilon}^{a}$ are $\epsilon$-approximations of the wet and the dry part of the solid

$$
\begin{gathered}
\partial \Omega_{\epsilon}^{r} \simeq \partial \Omega \cup \partial_{S} \omega \quad \text { (receding contact zone) } \\
\partial \Omega_{\epsilon}^{a} \simeq \partial \Omega \backslash \partial_{S} \omega \quad \text { (advancing contact zone). }
\end{gathered}
$$

\section{Numerical implementation}

The first issue is the choice between a penalty formulation to implement the volume constraint and the use of a Lagrange multiplier. We opted for a Lagrange multiplier because of its physical meaning associated with Laplace law (2.4). So, the Euler-Lagrange equation for the phase field model becomes (here we set $G=0$ and $a=1$ for simplicity)

$$
\begin{cases}-\epsilon \triangle \phi+\frac{1}{\epsilon} \phi(1-\phi)(1-2 \phi)+\lambda=0 & \text { in } \Omega \\ \phi=\phi_{S} & \text { on } \partial_{S} \Omega \\ \phi=0 & \text { on } \partial_{V} \Omega\end{cases}
$$

where the value of $\lambda$ has to be calculated in order to match the constraint $\int \phi=\mathcal{V}(t)$.

To solve the equilibrium equation we transform the problem into a parabolic PDE generated by a gradient flow [5], and we follow an artificial relaxation dynamics until the system reaches the equilibrium configuration. The gradient flow is introduced by setting $\phi=\phi(\tau, x)$, where $\tau$ is a fictitious time, and solving

$$
\phi_{\tau}=\epsilon \triangle \phi-\frac{1}{\epsilon} \phi(1-\phi)(1-2 \phi)-\lambda .
$$

Here the Laplacian is with respect to the space derivatives and the subscript $\tau$ denotes a time derivative. The solution of our original equation (5.1) will be $\lim _{\tau \rightarrow+\infty} \phi(\tau, \cdot)$. In fact, along the flow (5.2), the energy is decreasing in time

$$
\frac{\mathrm{d}}{\mathrm{d} \tau} E_{\epsilon}=-2 \int_{\Omega}\left|\phi_{\tau}\right|^{2} \mathrm{~d} x \leq 0 .
$$

For the time integration of the equation, we have found it convenient to use a forward Euler scheme. Of course, like for the standard heat equation, this scheme is only stable for small values of $\mathrm{d} \tau$. It is well known that treating the Laplacian term as implicit allows for bigger values of $\mathrm{d} \tau$ at the expense of solving a linear system at each iteration. Treating the entire right hand side (Laplacian and non-linear term) as implicit gives an unconditionally stable scheme, but requires solving a system of non-linear equations. We have tested all these schemes and we have found no significant advantage of the implicit over the explicit algorithm. Also, since we are only interested in converged (in the fictitious time $\tau$ ) solutions, there is no need to use higher order (semi-implicit) methods like Cranck-Nicolson.

In order to find at each iteration the correct value for the Lagrange multiplier associated with the volume constraint $\lambda$, we have used a splitting method. Namely, given an initial guess $\phi^{0}$ that satisfies the appropriate 
boundary condition (for the Dirichlet case), we follow the scheme

$$
\begin{aligned}
& \phi^{N+\frac{1}{2}}=d \tau\left(\epsilon \triangle \phi^{N}-\frac{1}{\epsilon} \phi^{N}\left(1-\phi^{N}\right)\left(1-2 \phi^{N}\right)\right)+\phi^{N} \\
& \lambda^{N}=\frac{\mathcal{V}-\int_{\Omega} \phi^{N+\frac{1}{2}}}{\int_{\Omega} 1} \\
& \phi^{N+1}=\phi^{N+\frac{1}{2}}+\lambda^{N}
\end{aligned}
$$

where $\mathcal{V}=\int_{\Omega} \phi^{0}$. By construction, we have that $\int_{\Omega} \phi^{N}=\mathcal{V}$ stays constant during the iterations. (For semiimplicit or fully implicit schemes, the same method applies with only slight modifications.)

For the space derivatives we have used instead a high order approximation nine-point stencil. This is classical in the 2-D case, but non trivial in the axisymmetric case. The derivation can be found in [16].

When studying the hysteresis of the contact angle, we notice that the conditions (2.12) and (3.8) derived for the limit configurations as $\epsilon$ goes to zero do not apply exactly in the approximate problem we solve (this is the meaning of the " $\simeq$ " symbol in (4.15) and (4.14)). Indeed we have to consider that the interface is not yet sharp, but has a width which depends on $\epsilon$. The effect of this is that our drops tend to advance or recede quicker than real ones. In order to stabilize the triple junction among the phases we add a third zone on the solid surface which is in contact with the liquid-vapor interface (see Fig. 4). On this third zone we put a boundary condition that correspond to a bigger advancing contact angle near the free area and a condition for a smaller receding angle near the wetted zone. The precise value of these stabilizing angles are specified in the example section.

\section{EXAmples}

In this section we describe some numerical simulations. Our goal, here, is to show how rich a behavior can be captured in spite of the simplicity of our model. The spirit of this demonstration is that of a proof of principle, and we present the results of "small simulations". Our efforts towards devising numerical efficient schemes in order to make large scale simulations possible will be described elsewhere.

The computational grid is of $100 \times 100$ points regardless of the physical size of the objects we simulate. With such a grid, we can set $\epsilon=0.008 \cdot a$ where $a$ is the coefficient in the potential (2.10). This ensures interfaces of width $\simeq 5$ grid points: a value small enough with respect to the size of the drop, but sufficient for a good resolution of the shape of the interface.

The value of $\mathrm{d} \tau$ depends on the evolution scheme. For the forward Euler scheme we set $\mathrm{d} \tau=0.001$, while we can reach $\mathrm{d} \tau=0.005$ for the semi-implicit scheme. Several tests proved the stability of the simulation with these values.

In the 2-D simulations we use a trapezoidal rule for the quadrature, while we pass to the Cavalieri-Simpson rule in the axisymmetric cases. We followed [18] for the condition on the axis of symmetry in order to ensure a second order accuracy. The convergence of the schemes is tested on the time gradient. We always obtained a value for this estimator below $10^{-9}$ summing the contribution of all computational nodes.

A remark about the size of the simulated drops is in order. If we set gravity to zero, the capillary problem is purely geometric and the length scale is irrelevant. In a realistic situation gravity can never be zero, but there is a typical length above which its effects become noticeable. We can define this capillary length, $\kappa^{-1}:=\sqrt{\sigma_{L V} / \rho g}$ (usually, for water in contact with air, it is of the order of few $m m$ [9]). If the size of the drop is much smaller than this value, then capillary forces dominate gravity. Thus, setting $G=0$ means to consider very small drops. When gravity is non zero we will specify precisely the length scales for which the simulation is relevant.

\subsection{Spherical cap}

We consider a drop on a plane and we first increase and then decrease its volume in order to observe the hysteresis of the contact angle. This simulation is performed in order to obtain a benchmark case. Indeed, the analytic solution for this problem is known: it is a spherical cap that does not move its base 


$$
\frac{52 D}{21}
$$



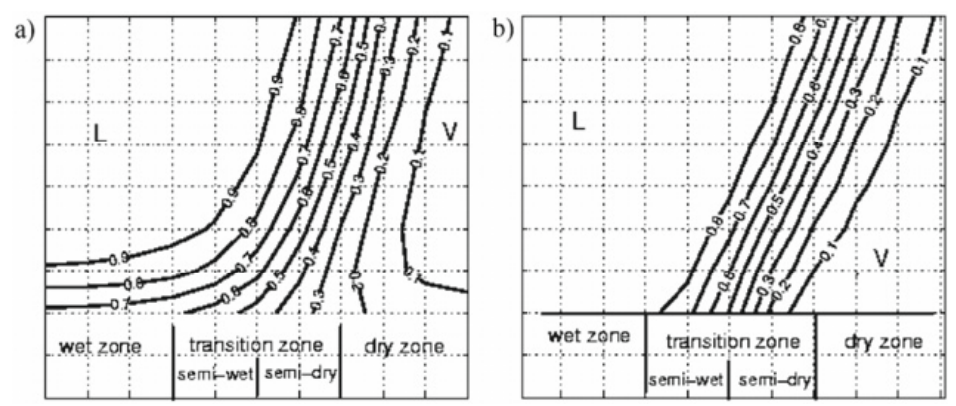

FIGURE 4. The determination of the different zones on a solid surface modeled by Dirichlet BC (a) and Neumann BC (b).

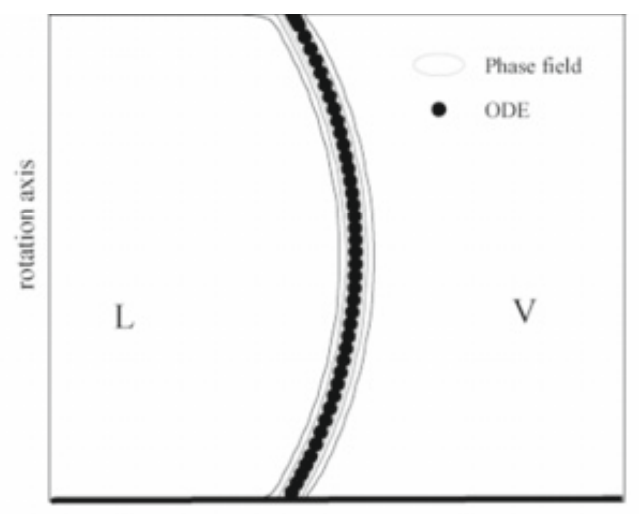

FigURE 5. We check the initial position of the phase field solution with the shape given by the integration of the ODE arising from the sharp interface formulation.

We proceed as follows, on the row of computational nodes above the solid, we find the transition point between values smaller or larger than 0.5 and we project this point on the solid. The semi-wet zone (respectively semidry) consist of the two nodes from this point towards the liquid (toward the vapor). We reached the desired stabilization decreasing (increasing) by 15 degrees the angle that the Neumann boundary condition would impose in those points. For the Dirichlet scheme we need a modification of 20 degrees. We summarize the situation with a graph in Figure 4, where we plot the contour lines of a drop with a Young contact angle of 120 degrees. The algorithm proceeds as described in Section 4: starting from the solution at time $t$, we identify the three zones on the solid surface; we then calculate the solution at time $t+\delta t$ (i.e. with an increased or decreased volume) keeping fixed the boundary conditions calculated from the previous step. For this procedure the Neumann scheme is more precise, because the contour lines are not bent by the solid surface. This explains the improvement passing from panel a) to panel b) in Figure 3.

Because of these results, in the next sections we will use the Neumann scheme each time we deal with hysteresis. The Dirichlet formulation will be used when we deal with non-smooth geometries for the solid (i.e. when the normal derivative cannot be directly defined) and dissipation effects are not our main interest.

\subsection{Drop between parallel plates}

Inspired by the striking experiments of Lafuma and Quéré [12], we consider the case of a drop compressed between two plates. 


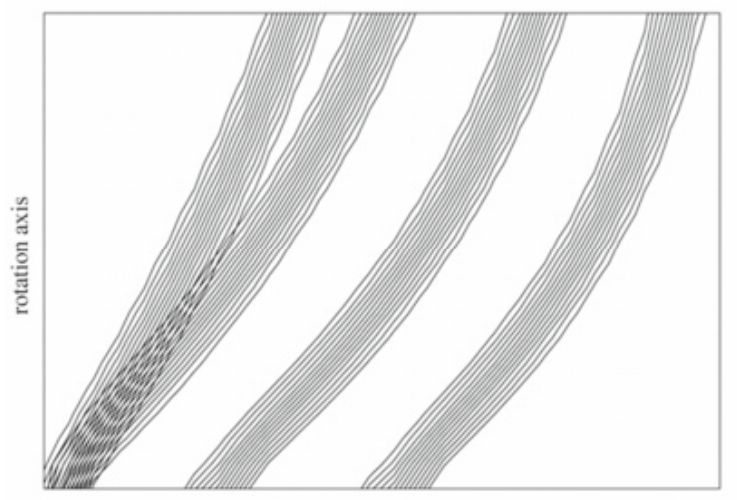

FiguRE 6. Evolution of the front near the lower plate during compression; notice the initial modification of the contact angle, while it remains fixed in the following steps.

Our results are plotted in Figures 5 and 6 . What we can observe with these simulations are the macroscopic effects of hysteresis. The physics at the micro (nano) scale is explored later, when we consider textured solid surfaces (pillars).

Energy minimizers are again axisymmetric. Figure 5 compares the phase field solution with the numerical solution of the ODE arising from the sharp interface formulation. We stick to an axisymmetric formulation also for the evolution problem.

The procedure followed for the quasi-static movements of the plates is based on a "predictor-corrector" scheme. Starting from a stable state, we cancel (add, respectively) a computational row at half the distance from the plates in order to decrease (increase) the distance between the plates. The prediction stage is done by looking for a new energy minimizing configuration, but keeping artificially fixed the wetted zones over the two plates. In the correction stage we release this constraint as follows. If the equilibrium configuration of the prediction stage has reached or even overcome the advancing (receding) contact angle, then a new minimization is performed. Otherwise the previous shape is accepted (see Fig. 7). In this way we do not loose information about the liquid-solid interface that are essential in the modeling of hysteresis of the contact angle.

Figure 6 collects a sequence of snapshots of the evolution of the profile near the lower plate: we plot the contour lines of $\phi$ at several time steps during the simulation, superimposing them. The picture shows clearly the initial variation of the contact angle and the subsequent advancing of the front with a constant contact angle equal to the advancing angle.

\subsection{Drops on an inclined plane: the 2-D case}

The 2-D simulation represents a portion (of unit thickness) of a 3-D situation invariant in the orthogonal direction. This, admittedly artificial, scenario of a cylindrical drop is already quite rich and interesting. In Figure 8 a typical situation is shown: the hysteresis of the contact angle allows for the equilibrium of a drop on an inclined plane.

In the simulations we change the inclination of the plane and we follow the changes in shape up to the limit configuration in which the drop starts to move and roll down. In Figures 9-12 the effect of gravity on drops of different volumes becomes more and more visible as the size of the drops increases. In these graphs we only draw the contour line $\phi=0.5$ and we superimpose the lines coming from different inclinations of the plane.

We consider water drops in air $\left(\sigma_{L V}=73 \mathrm{mN} \cdot \mathrm{m}^{-1}\right.$, hence $\left.\kappa^{-1}=2.7 \mathrm{~mm}\right)$ on a hydrophobic solid with $\theta^{Y}=120^{\circ}$; the contact angle hysteresis is set to $\pm 15^{\circ}$.

Like in a laboratory experiment, the initial condition for the simulation is important: with a first calculation we find the drop of the desired volume in the absence of gravity and meeting the Young angle condition (dotted line in the pictures). Then gravity and dissipation are added at the same time. As a consequence, the drop 


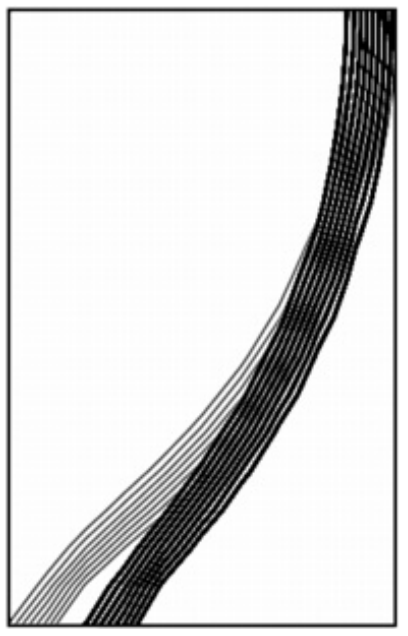

FIGURE 7. An advancing drop: the lines starting nearer to the bottom left corner represent the rejected configuration in which the contact zones between liquid and solid plates are artificially pinned.

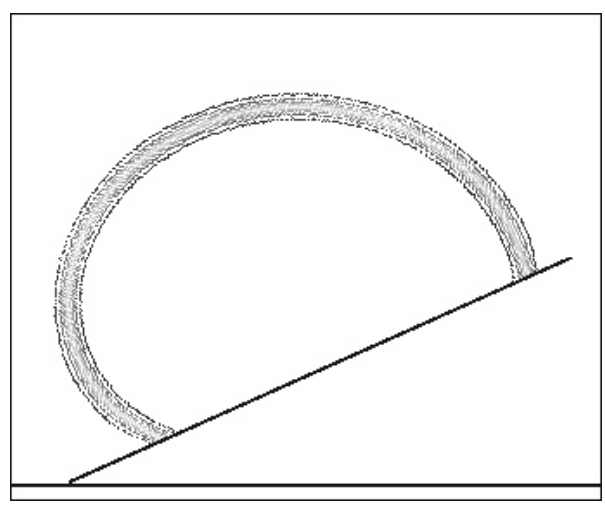

Figure 8. A phase field representation of a drop in equilibrium on an inclined plane. Equilibrium is an effect of the hysteresis of the contact angle.

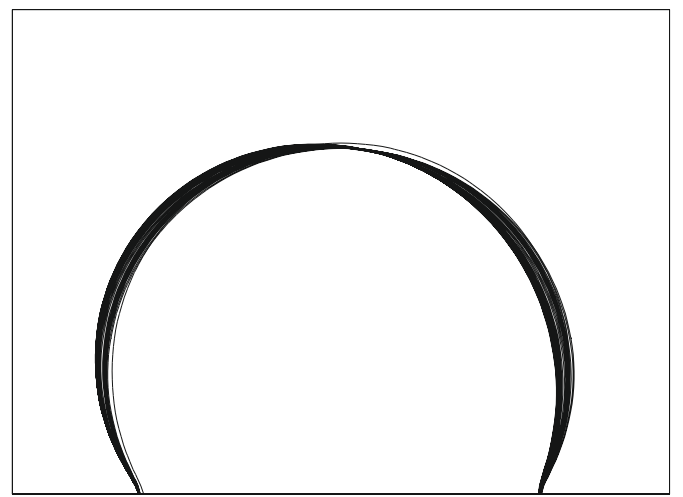

Figure 9 . For $\bar{d}=2.2$, the drop is stable up to $90^{\circ}$. 


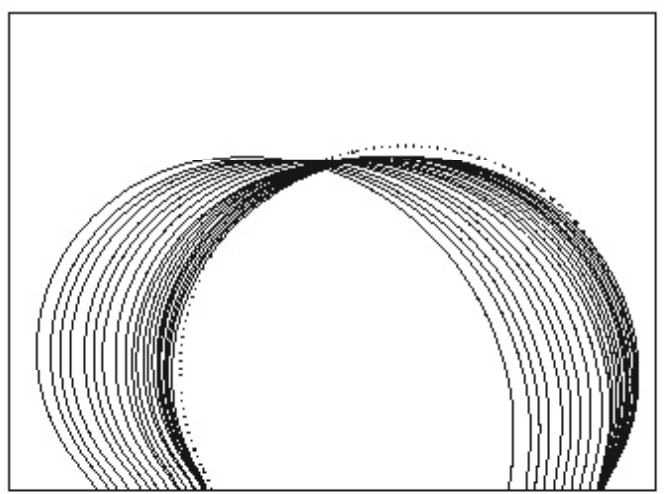

Figure 10. For $\bar{d}=3.6$ the drop is stable up to $60^{\circ}$.

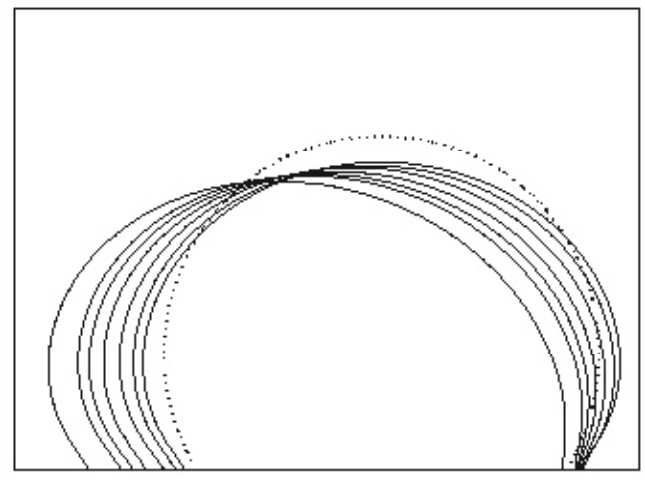

Figure 11 . For $\bar{d}=5.8$ the limit of stability is $18^{\circ}$.

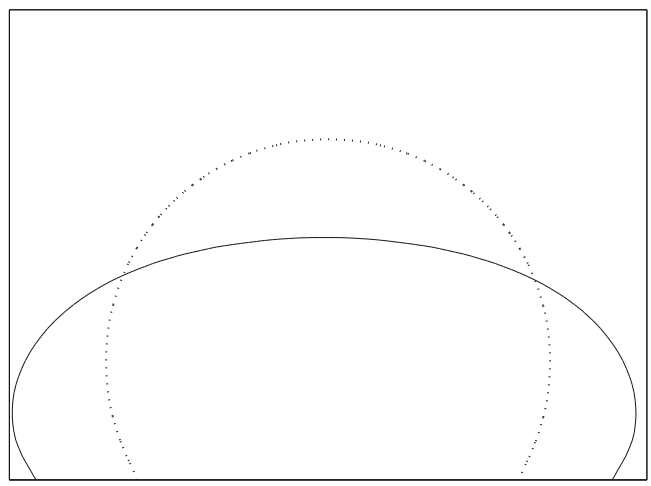

Figure 12 . For $\bar{d}=10.9$ the drop falls already at $3^{\circ}$.

stays symmetric but it acquires a contact angle larger than the Young value. The bigger the volume, the higher is the gravitational effect and thus the wider the angle. The effect of this is also visible when we later incline the plane: if the size of the drop is above the capillary length, the drops change their shape and their wet zone. With this preparation, drops move first the advancing zone: indeed the starting angle is closer to the advancing angle and the drops will reach it sooner. 


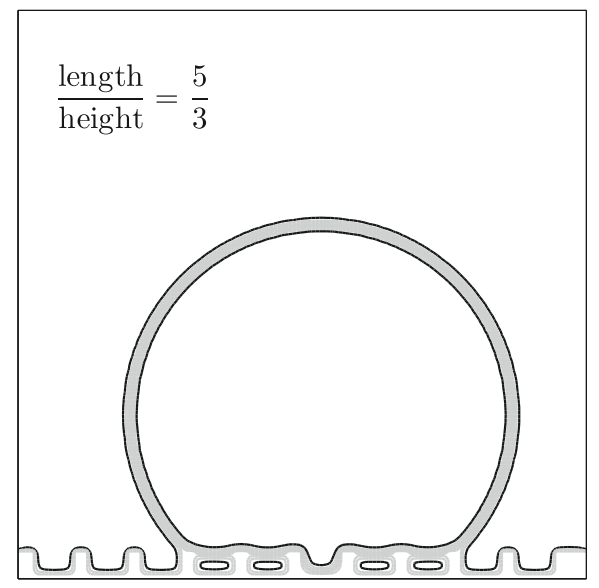

Figure 13. In the initial configuration the drop fills the central hole, but it can jump to the next pillars.

We increase the inclination of the plane slowly in order to simulate a quasi-static evolution of the type described in Section 3. At each step we increase the angle by $3^{\circ}$. In the pictures we superimpose the equilibrium configurations, as long as they exist. This means that, after the last frame shown, the drop falls down because gravity overcomes dissipation and the quasi-static model cannot describe what is happening next.

In order to compare our results with the results of 3D experiments, we consider an auxiliary parameter. We define $\bar{d}$ as the diameter of the sphere whose volume is equal to that of our cylindrical drop with unit height. The pictures show that for values less than the capillary length the gravity effects are not visible. For $\bar{d} \simeq \kappa^{-1}$ something moves, but the detachment is not apparent. Above this critical size the drops are flattened by gravity, they fall earlier, and with a smaller inclination of the plane.

\subsection{Pillars}

Wetting phenomena on a (microscopically) rough surface have been the object of intense recent studies (see [9] and the references therein). Two "models" are used to interpret the experimental evidence: the Cassie-Baxter and the Wenzel one. In the first one, the main assumption is that the liquid phase sees only the top of the asperities of the solid phase, leaving some vapor in the holes under it. The Wenzel model is based on the opposite scenario: the liquid fills all the cavities of the solid. This difference produces different predictions on the advancing and the receding contact angle.

Here we propose some simulations in a 2D geometry that illustrate some aspects of the phenomena described above. In Figures 13 and 14 a drop is placed over a periodic array of pillars. Increasing the drop volume, the liquid can fill the holes or it can jump them. For $\theta^{Y}=120^{\circ}$, theoretical predictions based on homogenization theory [1] say that absolute minimizers will jump if $a / b \leq 2$, where $a$ is the width of the hole and $b$ its height. Our results are very close to that value. The slight disagreement is explained by the fact that an advancing drop with a thick interface "foresees" the contact with the next pillar.

An important remark is the following. Our current implementation of the method limits us to "small" simulations. This forces us to work with drops of a size comparable to that of the pillars. This excludes from our results the analysis of the implications on the behavior of drops of macroscopic sizes, because the size of our pillars is typically few nanometers. In spite of these limitations, we can still reproduce a very rich range of interesting physical phenomena, related to stability and metastability of capillary drops on rough surfaces. A first interesting situation is the one described by Lafuma and Quéré in [12]: even in a geometry where the solid roughness would produce a Cassie-Baxter energy minimizing state, a Wenzel state can be reached by imposing 


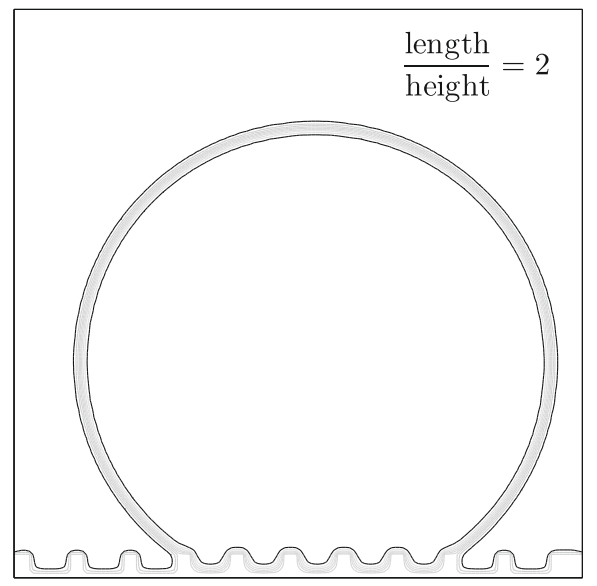

FIGURE 14. By slightly decreasing the height of the pillars the situation changes completely.
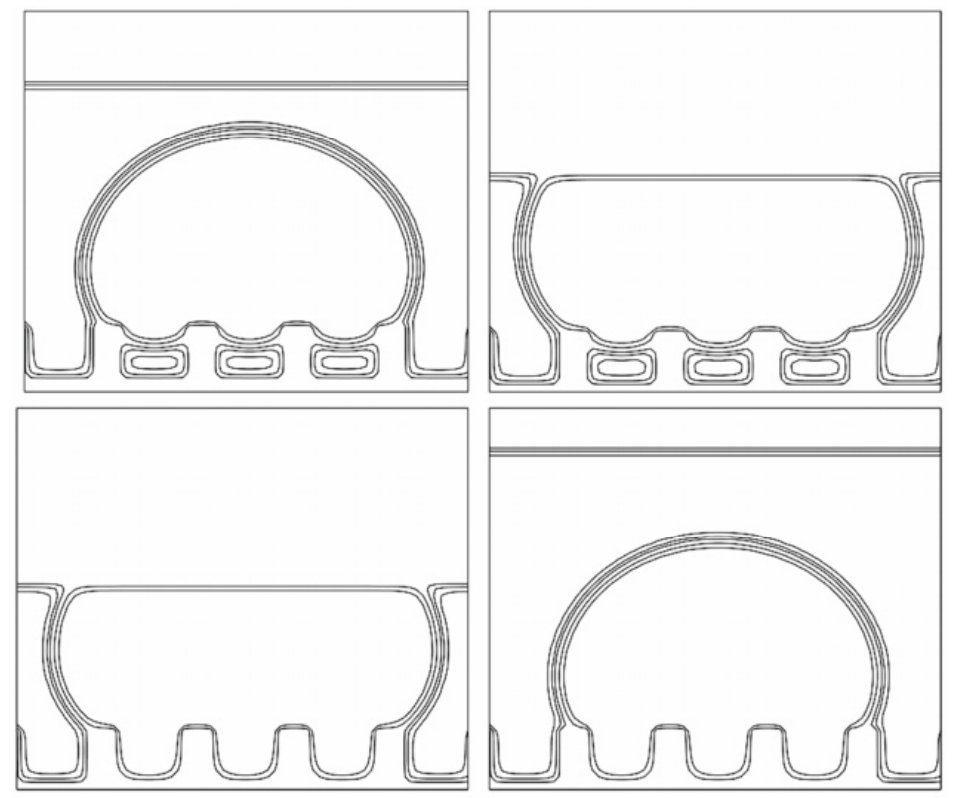

Figure 15. Compression induces a meta-stable Wenzel state on a Cassie-Baxter drop.

an external force. Moreover, when this force is relaxed, this final state is maintained, reflecting its (meta)stability. For large drops, gravity is a strong enough force to produce this effect, while the technique of the squeezing plates can achieve the same goal for any drop size. The results obtained by this second "technique" are shown in Figures 15 and 16. In the first group of pictures the upper plate is flat, while in the second group it is rough. Both situations allow for the transition between the two regimes, but the final configuration is different: drops adhere stronger on rough surfaces (if they are in a Wenzel regime [12]) and the strength of this bond can even allow for a splitting of the drop.

Another interesting experiment is described by Callies and Quéré in [7]. They put a large drop on a rough surface that admits as ground state the Wenzel one. But the small curvature of such a large drop allows to observe a metastable Cassie-Baxter state. They let the drop evaporate and when a critical size is reached, 


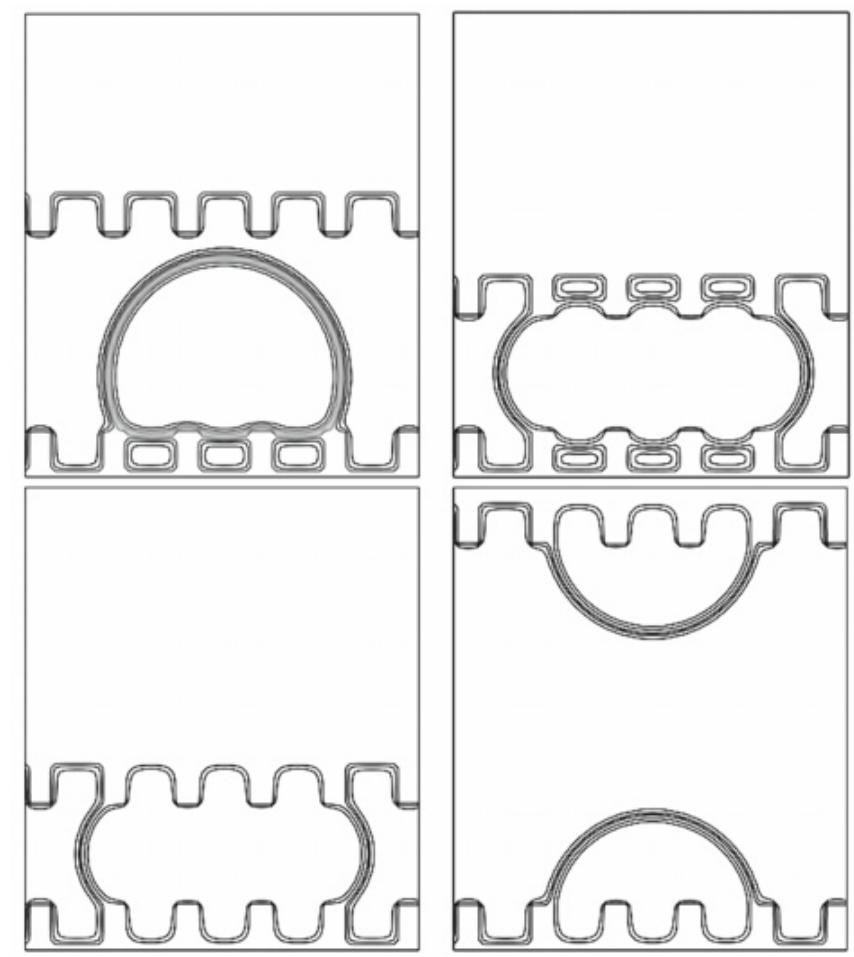

Figure 16. Compression between two rows of pillars. Notice the splitting of the drop.

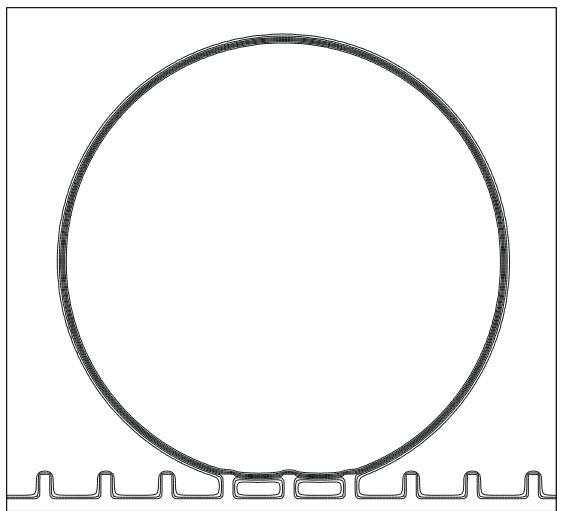

FiguRE 17. With a low curvature we can observe a C-B metastable state.

a sudden change in the shape is observed: due to the larger curvature of the smaller drop, the water fills the solid roughness and a large change in the contact angle is produced. In Figures 17 and 18 we show a numerical version of a very similar experiment. 


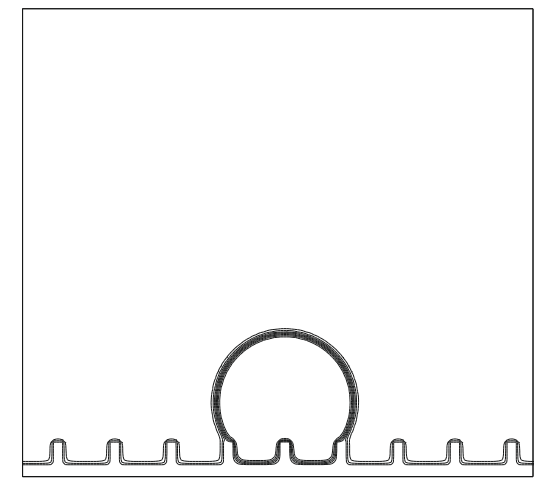

FIGURE 18. Decreasing the volume and so increasing the curvature, the Wenzel state is reached.

Acknowledgements. We gratefully acknowledge the financial support of the Italian INdAM "F. Severi" (Research Project "Mathematical Challenges in Nanomechanics") and of the EU through the Marie Curie Research Training Network MULTIMAT (Contract Number MRTN-CT-2004-505226).

\section{REFERENCES}

[1] G. Alberti and A. De Simone, Wetting of rough surfaces: a homogenization approach. Proc. R. Soc. A 461 (2005) 79-97.

[2] G. Alberti and A. DeSimone, Quasistatic evolution of sessile drops and contact angle hysteresis. In preparation (2009).

[3] G. Alberti, G. Bouchitté and P. Seppecher, Phase transition with line-tension effect. Arch. Rat. Mech. Anal. 144 (1998) 1-46.

[4] S. Baldo and G. Bellettini, $\Gamma$-convergence and numerical analysis: an application to the minimal partition problem. Ricerche di Matematica 1 (1991) 33-64.

[5] W. Bao and Q. Du, Computing the ground state solution of Bose-Einstein condensates by a normalized gradient flow. SIAM J. Sci. Comp. 25 (2004) 1674.

[6] A. Braides, $\Gamma$-convergence for beginners. Oxford University Press (2002).

[7] M. Callies and D. Quéré, On water repellency. Soft Matter 1 (2005) 55-61.

[8] G. Dal Maso, An introduction to $\Gamma$-convergence. Birkhaüser (1993).

[9] P.-G. De Gennes, F. Brochard-Wyart and D. Quéré, Capillarity and Wetting Phenomena. Springer (2004).

[10] A. DeSimone, N. Grunewald and F. Otto, A new model for contact angle hysteresis. Networks and Heterogeneous Media 2 (2007) $211-225$

[11] R. Finn, Equilibrium Capillary Surfaces. Springer (1986).

[12] A. Lafuma and D. Quéré, Superhydrophobic states. Nature Materials 2 (2003) 457-460.

[13] L. Modica, Gradient theory of phase transitions with boundary contact energy. Ann. Inst. H. Poincaré Anal. Non Linéaire $\mathbf{5}$ (1987) 497.

[14] L. Modica and S. Mortola, Un esempio di Г-convergenza. Boll. Un. Mat. It. B 14 (1977) 285-299.

[15] N.A. Patankar, On the modeling of hydrophobic contact angles on rough surfaces. Langmuir 19 (2003) 1249-1253.

[16] S.J. Polak, An increased accuracy scheme for diffusion equations in cylindrical coordinates. J. Inst. Math. Appl. 14 (1974) 197-201.

[17] P. Seppecher, Moving contact lines in the Cahn-Hilliard theory. Int. J. Engng. Sci. 34 (1996) 977-992.

[18] J.C. Strikwerda, Finite Difference Schemes and PDE. SIAM (2004). 